\title{
EXPANDABLE AND ROBUST LABORATORY FOR MICROPROCESSOR SYSTEMS
}

\author{
J-S. Chenard, A. U. Khalid, M. Prokic, R. Zhang, K-L. Lim, A. Chattopadhyay and Z. Zilic \\ Dept. of Electrical \& Computer Engineering \\ McGill University \\ Montreal, Quebec
}

\begin{abstract}
We present the design and use of McGumps laboratory kit for teaching microprocessor and embedded systems. The kit facilitates efficient learning of relevant hardware and software design techniques that will not get outdated soon. We describe the typical use of this kit in our courses.
\end{abstract}

\section{INTRODUCTION}

Widespread use of microprocessor systems and the rapid introduction of new processors, computer interfaces and communication standards requires state-of-the art laboratory teaching practices that elude obsolescence. McGill University Microprocessor Systems kit McGumps was designed to be expandable and to facilitate complex teaching goals. So far, four different boards were designed, with one new board added each semester for course projects.

While designing the laboratory equipment, we wanted to achieve relevance, possibility to generate a large set of exercises, including complex SW/HW projects, as well as overall robustness and ease of use. In prior years we have taught using several different processor architectures. Most often, existing kits are either simple and based on outdated processors (e.g., 8-bit MCUs), or modern, but complex to use and debug. In the end, we decided to build our own kit, using the MSP430 microcontroller family from Texas Instruments. This family is modern, yet simple enough for teaching purposes. In addition to its RISC instruction set, in-system debugging and Flash programming capabilities, it also contains a good sample of typical hardware modules: UART, SPI, A/D converter, timers, watchdog timer, PWM output, user-code Flash programming and vectored interrupts. This set of features is universal to many microprocessor families from various vendors. Students can also learn how to design systems using a variety of energy-saving modes, which is a feature where this processor excels. Possibility for debugging code without using complex and expensive in-circuit emulators makes this processor especially suitable for teaching.

\section{KIT DESIGN}

In the early stages of design, we have decided to include a CPLD in addition to the CPU. Such device allow a large variety of lab experiments to be set up without adding additional hardware. To provide simple and flexible programming and debugging, we have built in both the JTAG programmers i.e., Texas Instrument FET and Altera CPLD Byteblaster MV. Re-programming the CPU or the CPLD only requires a $\mathrm{PC}$ with a parallel port. These programmers are compatible with virtually all design software, and both CPU and CPLD can be re-programmed without any jumper changes on the board.

Features on the main board also include:

- 1 parallel and 2 EIA-232 compatible interfaces

- Dallas i-Button connector

- MMC connector

- 2-digit LED display

- Serial loader for the MCU as an alternative to JTAG

- $0.1 "$ spaced prototyping area

- 40-pin keyed connector for CPU expansion ( LCD screen, motor control, RF communication )

- 40-pin header for wirewrapping to the CPLD for student designs and expansion

Both expansion connectors feature $5 \mathrm{~V}$ and $3.3 \mathrm{~V}$ power rails allowing single cable attachments to expansion cards. The main expansion connector is keyed to eliminate errors in attaching the cable. The 40-pin connector is the same as PC ATA connectors, providing a low-cost source of replacement parts.

\subsection{1. $C P L D$}

The CPLD allows experiments in which students build their own peripherals for good understanding of processor interfacing. The CPLD is connected to the processor using both dedicated and shared signals. We have found that the early labs designed for resolving conflicts on shared lines, prepared students for the course project and real-life scenarios involving many current interfacing standards. The Altera $\mathrm{CPLD}^{1}$ and related tools (Altera and ModelSim) were chosen for compatibility with earlier courses at McGill. The CPLD also acts as a $5 \mathrm{~V}$ to $3.3 \mathrm{~V}$ voltage level converter allowing the use of $5 \mathrm{~V}$ peripherals to be connected to the low-power CPU.

The board can be powered through four different power sources. A wall-mounted transformer, direct soldered connections and spring loaded connectors provide the first set of power sources. An alternate power source is the PC parallel port when used as a JTAG programmer. The parallel port power source provides limited current, that suffices when the CPLD is de-activated. This allows students to program and debug their initial experiments using their home computer without having to carry power sources or battery packs. Power is provided by a low-drop voltage regulator, a protection diode and a self-resettable fuse. This setup protects the board from accidental power reversal and virtually

\footnotetext{
${ }^{1}$ We acknowlege the support of Altera Corporation.
} 
eliminates its downtime in the lab. Students at most experience a 15 second reset, after which the board continues with their program.

\subsection{Peripheral Boards}

In addition to the main board, each year a new peripheral module is being developed in front of students, and deployed for the final project. In past, students were only peripherally involved in that design, but the level of their participation is increasing commensurate with our confidence in the project success.

McGuld is an expansion board containing a $128 \times 64 \mathrm{LCD}$ with integrated controller and a touch panel. The main purpose of this board is to let students design user interfaces, while learning about low-level interfacing for touch screen LCDs, as well as RAM constraints and real-time operation of a MCU with limited resources. We have actually built two versions of the touch screen controllers. In some boards, we have built a purely MOS-based circuit that relies on the CPU's A/D converter to provide the pen position. For other boards, we have built in a modern integrated touch screen controller with interrupt capability and low-power modes.

The McGuld boards additionally include either serial flash memory or EEPROM ICs that share the same SPI bus as a touch screen controller. Students needed to learn how to address multiple slave devices on a shared bus in order to fully utilize the board and add features to their project. In selecting the customized version of McGuld, students have to make an early a decision on which interfaces they wish to use and this further motivated them to study the given technologies before committing to their use.

$M c Z i g$ is a wireless expansion module based on the Chipcon CC2420 802.15.4 2.4GHz integrated radio transceiver. The board features an integrated printed antenna, removing fragile parts and providing a low-cost module. The wireless board emits low energy and in the ISM band, eliminating the need to seek a license for its use.

$M c Z u b$ is a stand-alone USB-enabled board that features the Chipcon CC2420 chip and an ARM-based Philips LPC2106. With more memory and computing power than MSP430, the device allows students to experiment with complex network topologies. The USB can be used for data communication or firmware upgrades. The board can be powered through USB or via an external adapter.

\subsection{Testing}

Since the hardware was developed in-house, test firmware and procedures were put in place for technicians to maintain the hardware. The hardware design includes ESDprotection diodes on sensitive elements such as MOS transistors present on the McGuld board, providing enhanced reliability. Students are thus ensured that the equipment is in proper working condition. Using the reference test code, the TAs can prove to students that problems are due to their implementation and not a board failure, providing a fast unambiguous answer.

All the schematics, bill-of-materials, part numbers and suppliers are available on-line, ensuring a long lifespan for the teaching material. Special attention was given to the CPLD - the device is socketed since it is the most failureprone due to its interface with student-designed hardware. Since the Gerber files for PCB production are also under the university control, extra blank PCBs were produced in the event students wish to get a permanent version of their projects for research or other uses. In addition, other members of the faculty have made use of the centralized system to introduce the hardware to other courses, for example Real-Time Embedded Systems.

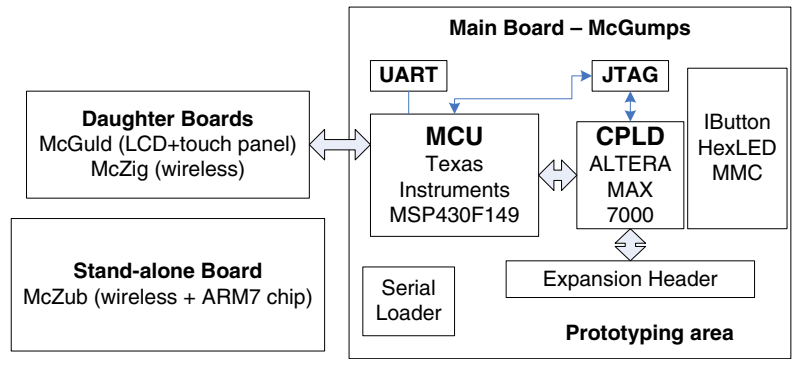

Figure 1. System Overview

\section{TEACHING METHODOLOGY}

Labs are set up to introduce the McGumps features in a progressive and structured fashion. The initial lab familiarize the students with assembly language programming, the development environment and makes use of the instruction set simulator. A small project, such as implementation of a Babagge machine or a small arithmetic computation is given and validated using a set of automated test routines by the TA during the demonstration. The second experiment requires student to interface "C" code to their assembly code. In this lab, the students have to download their executable file to the McGumps board and perform the demonstration using the real hardware. In the third lab, students are asked to produce drivers for the USART, using a scenario involving user interaction. Keypads scanning methods, CPLD driven 7-segment LED drivers and timers are typical elements introduced at this stage. In the fourth lab additional drivers are required including SPI, touch-screen drivers and user-level flash programming, configuration storage and retrieval. Emphasis is put on running all the peripherals simultaneously and message-queues structure is proposed as a solution to parallelize various elements of the system. Some advanced teams may choose at this point to use a small realtime operating system such as $\mu \mathrm{C} / \mathrm{OS}-\mathrm{II}$ by Micrium.

A one-month final project completes the lab course. Students are given high-level specifications and must then choose from various alternative options which they wish to implement. Student progress is closely followed - a midproject review is carried to ensure that all the drivers development is going well, followed by a final evaluation of their work and a peer review by the team members. With this kit, students are able to finish ambitious projects that employ multiple components and have practical real-world application. Some recent examples include networked filesharing appliances, PDAs with a touch screen, handwriting recognition and secure wireless communication RSA.

We have been conducting surveys regarding its robustness, ease of use and usefulness in future microsystems endeavors [1]. The surveys and formal evaluations shows that the lab provides an excellent learning experience. Further, $90 \%$ of students find the equipment and supporting documentation to be adequate and robust, with the residual problems mainly due to the ad-hoc in-house soldering of small quantity boards, instead of the assembly through professional PCB services.

\section{REFERENCES}

[1] http://www.macs.ece.mcgill.ca/ mproki/ micropevaluation.html 